\title{
Diseño de un sendero agro ecoturístico en forma circuito con base en los potenciales naturales y productivos del Centro Experimental ICIDRI Masatepe de la Universidad Politécnica
}

\section{Agro-ecotourism trail design with circuit shape based on natural and productive potentials at the ICIDRI Masatepe Experimental Center, Polytechnic University}

\author{
Yaritsa Yanira González García ${ }^{1}$, Lucia Raquel Vado González ${ }^{1}$, Emelina Tapia Lorío ${ }^{2}$, Rosa María Reyes Pérez ${ }^{3}$ \\ ${ }^{1}$ Ingeniera Forestal graduada en la Universidad Nacional Agraria, Managua, Nicaragua \\ ${ }^{2}$ MSc. en Manejo y Conservación de los Recursos Naturales: ORCID: https://orcid.org/0000-0002-9837-7935 / emelina.tapia@ci.una.edu.ni \\ ${ }^{3}$ Lic. en Biología, ORCID: https://orcid.org/0000-0003-3342-6131/mreyes@ci.una.edu.ni \\ Universidad Nacional Agraria - Facultad de Recursos Naturales y del Ambiente
}

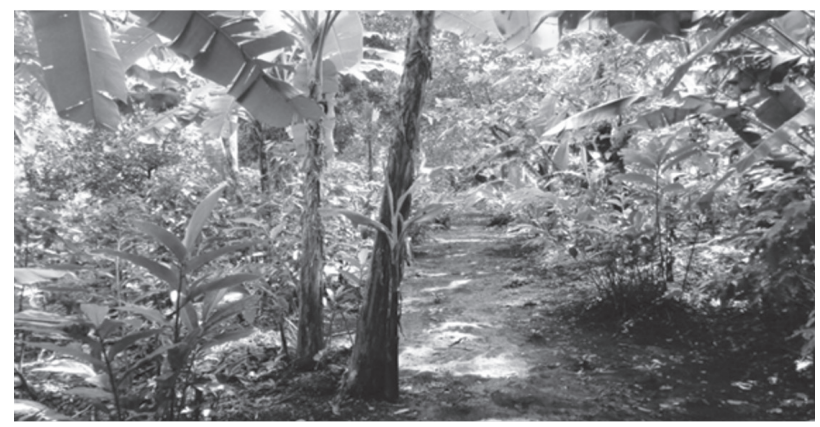

\section{RESUMEN}

El agroturismo en Nicaragua es una actividad teórico-práctica que se realiza en zonas o fincas con enfoque productivos (sean agrícolas o agropecuarias) y/o con ambientes naturales (bosques) de manera sostenible con el fin de generar productos de origen agrícola, pecuario, o forestal al usuario y propietario que lo desee. En este sentido, hace falta integrar este tema en espacios con características similares. Este estudio se realizó en el Centro Experimental del Instituto de Capacitación e Investigación en Desarrollo Rural Integral ubicado en la ciudad de Masatepe, departamento de Masaya, Nicaragua, propiedad de la Universidad Politécnica de Nicaragua, con el objetivo de realizar un diseño de un circuito agro ecoturístico sobre la base de los potenciales naturales y productivos del mismo. Se contemplaron tres etapas: (I) La de planeación y organización que facilitó a través de una visita previa, los tiempos y formas de recabar la información requerida; (II) La de trabajo de campo en el que se registró la información en dos visitas al sitio para la delimitación del área, realizar un inventario florístico y de fauna, y de la propuesta de estaciones interpretativas; y (III), de análisis e interpretación de toda la información recabada en conjunto con el equipo de docentes de la Universidad Nacional Agraria, del Instituto de Capacitación e Investigación en Desarrollo Rural Integral en Masatepe y de la Universidad Politécnica de Nicaragua; se revisó la propuesta y fue aceptada. Como resultados tenemos: a partir de los escenarios encontrados, 11 estaciones interpretativas en un tiempo no mayor de tres horas, pudiéndose observar en cada una de ellas una diversidad de flora y fauna, también ofrecen una gama de actividades que promueven el contacto con la naturaleza. Palabras claves: estaciones, recorridos, visitantes, interpretación ambiental.

\begin{abstract}
Agrotourism in Nicaragua is a theoretical-practical activity that is carried out in areas or farms with a productive focus (whether agricultural or agricultural) and / or with natural environments (forests) in a sustainable way in order to generate products of agricultural or livestock origin, or forestry to the user and owner who wants it. In this sense, it is necessary to integrate this theme in spaces with similar characteristics. This study was carried out at the Experimental Center of the Institute for Training and Research in Integral Rural Development located in the city of Masatepe, department of Masaya, Nicaragua, owned by the Polytechnic University of Nicaragua, with the aim of carrying out a design of an agricultural circuit ecotourism based on its natural and productive potentials. Three stages were contemplated: (I) The planning and organization that facilitated through a previous visit, the times and ways to collect the required information; (II) Field work in which the information was recorded on two visits to the site to delimit the area, carry out a flora and fauna inventory, and the proposal of interpretive stations; and (III), the analysis and interpretation of all the information collected in conjunction with the team of teachers from the National Agrarian University, the Training and Research Institute for Integral Rural Development in Masatepe and the Polytechnic University of Nicaragua was achieved; the proposal was reviewed and accepted. As results we have: from the found scenarios, 11 interpretive stations in a time no longer than three hours, being able to observe in each one of them a diversity of flora and fauna, they also offer a range of activities that promote contact with nature.
\end{abstract}

Key words: Stations, routes, visitors, environmental interpretation.
Recibido: 31 de enero del 2020 Aceptado: 4 de mayo del 2020

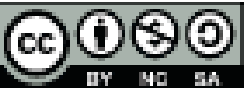

(C) pueden obtener en el correo edgardo.j
Copyright 2020. Universidad Nacional Agraria (UNA).
Los artículos de la revista La Calera de la Universidad Nacional Agraria, Nicaragua, se comparten bajo términos de la licencia Creative Commons: Reconocimiento, No Comercial, Compartir Igual. Las autorizaciones adicionales a las aquí delimitadas se pueden obtener en el correo edgardo.jimenez@ci.una.edu.ni 


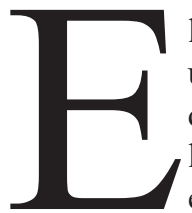

1 Centro Experimental Icidri Masatepe (CEIM), ubicado en Masaya, Nicaragua, es un centro que se dedica al cultivo de café bajo sombra y la conservación de los recursos naturales con enfoque agroecológico Instituto de Capacitación e Investigación de Desarrollo Rural Integral - Universidad Politécnica de Nicaragua (ICIDRI-UPOLI, 2011). Presenta potenciales naturales por su abundancia de flora y fauna y potenciales productivos por la diversidad de áreas cafetaleras y de otros cultivos; no obstante, la diversidad en cada caso y su estado es una debilidad debido a la ausencia y poca actualización de base de datos que requiere el centro convertirla en una oportunidad mediante la actualización de base de datos, entre ellos de un inventario que destaque sus aspectos ecológicos, técnicos y de proyección social; y mediante la elaboración de un diseño de circuito agro ecoturístico, poder ofertar a los usuarios su potencial natural y productivo.

En Nicaragua existen experiencias de estudios relacionados al ecoturismo y agroturismo. El ecoturismo se está desarrollando por iniciativas locales en áreas protegidas. Hay una serie de cooperativas, que están buscando cómo diversificar su actividad económica y están optando por el desarrollo turístico. El agroturismo en nuestro país se practica en zonas altamente productivas, ya sean agrícolas o agropecuarias. Estas zonas podrían pertenecer a una persona, cooperativa, o comunidades, pero deben integrar de manera sostenible las actividades, en las cuales se generan productos de origen agrícola, pecuario, o forestal según lo indica el Instituto Nicaragüense de Turismo (INTUR, 2009).

En este sentido, en Nicaragua hace falta integrar el tema del Agro ecoturismo, dado que en muchas zonas del país se incluyen actividades agrícolas y pecuarias dentro de un medio natural. Basado en esta premisa se realizó esta investigación en conjunto con tres funcionarios del Instituto de Capacitación e Investigación en Desarrollo Rural Integral (ICIDRI), en el Centro Experimental Icidri Masatepe (CEIM) El cual tuvo como objetivo principal proponer un diseño de circuito agro ecoturístico con base en los potenciales naturales y productivos del CEIM.

\section{MATERIALES Y MÉTODOS}

El CEIM se ubica en La Meseta de los Pueblos en el municipio de Masatepe, en la parte sur del departamento de Masaya, Nicaragua, entre las coordenadas geográficas UTM X 0593588 Norte y Y 1317866 Oeste, a 51 kilómetros al sur este de la Capital Managua, con altitud de $455 \mathrm{msnm}$; cuenta con un área de siete hectáreas. La investigación es de tipo descriptiva no experimental con análisis cualitativo; la metodología aplicada es tomada y modificada de la Guía para el diseño y operación de senderos interpretativos propuesta por la Secretaría de Turismo de México (SECTUR
MX, 2004), con la modalidad turística, denominada Agro ecoturismo y desarrollo sostenible propuesta por Ramírez, (2015) y basado en las características del sitio, se utilizó la metodología de Vaca, (2001) para un sendero en forma circuito. Se aplicaron tres entrevistas no estructuradas para recabar aspectos relacionados con el entorno y experiencia con actividades agro-ecoturísticas. Para la identificación de la flora representativa se contó con el acompañamiento del técnico del CEIM y en colaboración del Ing. Claudio Calero de la Universidad Nacional Agraria (UNA). Para el inventario de la fauna silvestre se utilizaron tomas fotográficas y en colaboración del Ing Miguel Garmendia de la UNA. De esta manera, el estudio se desarrolló en tres etapas:

Etapa I: Planeación participativa. Esta etapa se sub dividió en cuatro sub etapas: (1) dos reuniones preliminares con el Instituto de Capacitación e Investigación de Desarrollo Rural Integral (ICIDRI) de la Universidad Politécnica de Nicaragua (UPOLI), para que estudiantes de ingeniería Forestal realizaran prácticas pre profesionales en su finca experimental de Masatepe en el año 2014; una vez realizado el contacto y con la aprobación de la realización de prácticas pre profesionales (PPP) en el 2014, se consideró la posibilidad de que este espacio diera lugar a dos trabajos de investigación; (2) recopilación de información secundaria sobre las temáticas encaminadas al senderismo, agro ecoturismo y circuito en centros de información tales como el Centro Nacional de Información y Documentación Agropecuaria (CENIDA), revisión del documento base Planificación estratégica CEIM (ICIDRI-UPOLI, 2011) y de documentos electrónicos varios; (3) tres visitas de campo al sitio con asesores y autoridades del ICIDRI (reconocimiento del sitio, recabar diversos datos de flora y fauna y para concretar el diseño del circuito); y (4) revisión e identificación de dos fases del esquema metodológico propuesto por la Secretaria de Turismo (SECTUR MX, 2004) de México, que son Planeación (I) y Diseño (II) de senderos interpretativos y de formatos pre elaborados de la guía para la elaboración del plan de desarrollo turístico según Blanco (2008).

Etapa II: Delimitación del área de estudio. La etapa II se subdivide en tres subetapas y corresponden a: (1) Elaboración del croquis con el programa Arc View 3.2, de toda el área de estudio incluyendo caminos y estaciones. (2) Inventario florístico de árboles mayores de $10 \mathrm{~cm}$ de diámetro a la altura del pecho (DAP) en un área total de 7 hectáreas $(10 \mathrm{mz})$ para actualizar la base de datos del CEIM. (3) Inventario de potenciales atractivos (naturales y productivos) encontrados en los caminos del CEIM aplicando la metodología de referida por López y Ráudez (2010) y en base a ellos enfocar el diseño o planeación de la propuesta del sendero con fines agro ecoturístico. 
Etapa III. Análisis e interpretación de la información. Se procedió a consolidar la propuesta con la asignación de los nombres de cada estación interpretativa; se realizó la descripción de sus potenciales; se diseñó el tipo de sendero y posteriormente se aplicó la metodología de análisis FODA; y se organizó la presentación del trabajo al equipo técnico del ICIDRI en Managua. Con los aportes se concretaron los diseños de mapas (mapas: del Centro y el de la propuesta de la ruta del circuito agro ecoturístico). El diagnóstico y análisis a partir de la matriz de FODA elaborada para el CEIM fue con el fin de optimizar el desarrollo y funcionamiento del centro tomando en cuenta estrategias básicas recomendadas. Finalmente, se presentó y avaló la propuesta consensuada del diseño del circuito agro ecoturístico al equipo técnico del CEIM

\section{RESULTADOS Y DISCUSIÓN}

Características del CEIM. Con la información proporcionada por Instituto de Capacitación e Investigación en Desarrollo Rural Integral de la Universidad Politécnica de Nicaragua (ICIDRI-UPOLI (2011), con el recorrido realizado en la finca y con las entrevistas informales realizadas a tres funcionarios del CEIM, se pudo corroborar que esta finca demostrativa, efectivamente, es una opción de fácil acceso y de promoción ambiental que permite desarrollar diversas actividades agro ecoturísticas para turistas interesados en la naturaleza a través de los potenciales identificados; y como valor agregado, el poder participar de manera activa en cada una de las propuestas.

Ramírez, (2013) indica que el agroturismo abre un espacio según tipologías turísticas potenciales que se desarrollan en el área rural. Sin duda, esta actividad ofrece un espacio para poner en práctica las reflexiones teóricas $\mathrm{y}$ académicas sobre los beneficios de visualizar el desarrollo rural dentro de un enfoque de territorio, recordar que la actividad rural va más allá de lo agropecua $\neg$ rio, valorar la importancia de los empleos e ingresos no agrícolas, y comprender la importancia de la identificación, caracterización, y preservación de los recursos y los activos locales específicos, para generar procesos de bienestar local (Riveros y Blanco, 2003). "La contribución e importancia del agroturismo y el ecoturismo radica en el potencial de esta actividad en los efectos multiplicadores" (Mérida, García, Vasquez y Oros, 2003, p. 189).

Según Redclift y Woodgate (1993), un agroecosistema, es aquel trozo de naturaleza, que puede ser reducido a una última unidad con arquitectura, composición y funcionamiento propios y que posee un límite teóricamente reconocible, desde una perspectiva agronómica, para su adecuada apropiación por parte de los seres humanos con los CEIM recursos naturales: agua, suelo, energía solar, especies vegetales y el resto de especies animales. Larios, Salmerón y García (2014), plantean que los sistemas manejados de manera diversificadas (sistema agroecológico) preservan o mejoran sus recursos naturales permitiendo su perdurabilidad.

En este sentido el CEIM, por tratarse de una finca que integra diversos agrosistemas, contar con inventarios de plantíos de café en cinco pisos, plantaciones de frutales y arbóreos (ICIDRI-UPOLI, 2011), debía contar con un inventario de flora actualizado y con un inventario de fauna silvestre como valor agregado. Se identificaron las siguientes especies de flora: Acacia amarilla (Senna siamea), Acetuno (Simarouba amara), Aguacate (Persea americana), Cedro real (Cedrela odorata), Ceiba (Ceiba pentandra), Chaperno (Albizia adinocephala), Chilamate (Ficus sp.), Falso roble (Tabebuia rosea). Entre las especies de fauna que se lograron identificar se mencionan: Ameiva undulata (Holcosus undulatus), Mano de piedra (Dermophis mexicanus), Tragavenado (Boa constrictor), Iguana verde (Iguana iguana), Zorzal (Turdus grayi), Sorococa, Porococa o Lechucita serranera (Megascops clarkii), Pájaro carpintero (Melanerpes hoffmannii), Salta piñuela (Anthracothorax prevostii), Ardilla chiza (Sciurus variegatoides) y Tlacuache (Didelphis marsupialis). Que sirven de referencia para futuras investigaciones que se deseen realizar in situ.

Inventario de potenciales naturales y productivos del CEIM. Se lograron identificar seis potenciales naturales y seis productivos en el CEIM, a los cuales se le asignó una propuesta de actividades con fines agro ecoturísticas (Cuadro 1), que podrían desarrollarse según los intereses de los visitantes. Los potenciales naturales presentan diversas oportunidades para realizar actividades recreativas y prácticas de campo

Cuadro 1. Potenciales naturales, productivos y actividades a realizar en el

\begin{tabular}{ll}
\hline Potenciales naturales & Potenciales productivos \\
\hline - Especies arbóreas & - Café \\
- Paisajes & - Musáceas \\
- Fauna & - Producción de miel de abejas \\
- Flora & meliponas \\
- Amplia belleza escénica & - Aguacate \\
- Vista panorámica & - Cítricos \\
& - Pitahayas \\
\hline Actividades propuestas a realizar & Otras actividades propuestas a realizar \\
\hline - Avistamiento de aves & - Prácticas de campo \\
- Observación de flora y fauna & - Corte de café \\
- Miradores & - Siembra en camas Biointensivo (área \\
- Interpretación ambiental & de ensayo) \\
- Paisajismo & - Elaboración de abonos orgánicos \\
- Fotografía & - Cosecha de cítricos (limones, \\
- Campamento & naranjas, mandarinas) \\
- Observación de un fragmento de & \\
bosque tropical de la zona & \\
\hline
\end{tabular}


que incluyen desde la observación de diversos paisajes, horas y períodos de avistamiento, que están en dependencia de la época en que se visiten, e identificación de formas de vida vegetal y los potenciales productivos que inducen a los visitantes nacionales y extranjeros a la actividad propiamente de observación de los procesos y/o a la realización de prácticas de campo en las áreas experimentales y en sus diferentes procesos productivos tales como: procesamiento del café orgánico (vivero, plantaciones, corte, lavado, despulpado, secado).

Con este tipo de inventario se puede lograr la vinculación entre los diversos atractivos con los intereses de los visitantes; y la finalidad como lo expresa Sáenz (2017), es motivarlos a que puedan apreciar la importancia de rescatar todo lo natural, lo auténtico del lugar, lo histórico-cultural para que el producto Agro Ecoturístico se convierta en algo extraordinario para el turista; y sobre todo con la riqueza inimaginable de la naturaleza en todo su esplendor.

Ruta del circuito agro ecoturístico. El CEIM cuenta con una entrada principal, que permite el control de la entrada y salida de los visitantes. En base a esta condicionante, el sendero agro ecoturístico propuesto es de tipo circuito. En la Figura 1, se presenta el mapa del recorrido del circuito agro ecoturístico diseñado para el Centro Experimental ICIDRI Masatepe con sus estaciones propuestas para la interpretación ambiental y actividades productivas que se pueden llevar a cabo. El circuito agro ecoturístico cuenta con una distancia total de recorrido equivalente a 1124 metros, para un total de dos horas y 20 minutos como tiempo promedio para recorrer las 11 estaciones propuestas; 10 de éstas se pueden visitar en la época seca y lluviosa y solamente la estación 4 podrá ser visitada durante la época seca.

Por las características de cada estación (descripción o atractivos), cinco de las estaciones (centro de información, procesos del café, muestrario de variedades del café, el ensayo y cinco pisos) ameritan ser visitadas con un guía del centro, es decir bajo la modalidad de guiado, por la complejidad de la información y prácticas que se pueden realizar; las otras estaciones pueden ser visitadas en ambas modalidades (auto guiado y guiado), según el interés del visitante.

Castillo (2017) y SECTUR MX, (2004), establecen que una ruta de circuito debe ofrecer una serie de placeres y actividades distintivos de la misma con un inicio (entrada) y un fin (salida). También debe presentar una imagen integral (plano o croquis) a partir de la complementariedad con las estaciones predefinidas entre sitios, servicios e infraestructura y tener como lenguaje lo comunicacional (acompañamiento técnico).

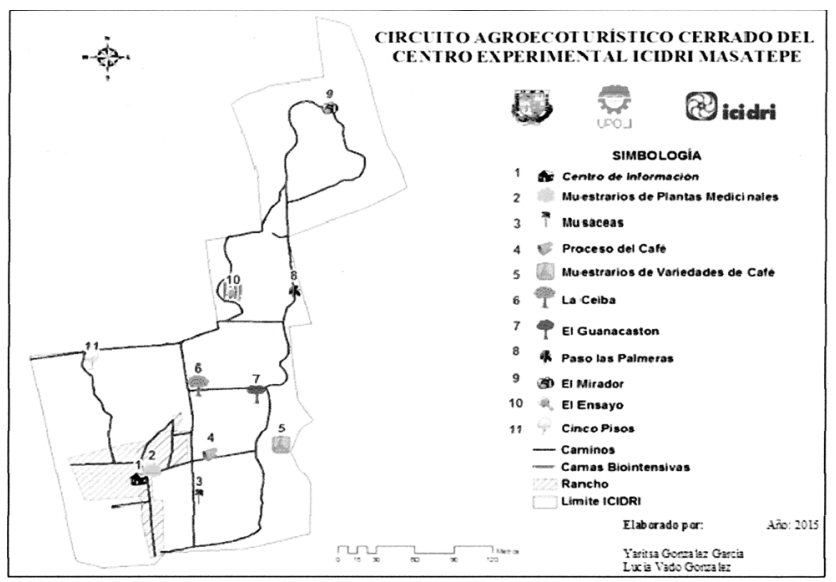

Figura 1. Mapa del circuito agro ecoturístico propuesto para el centro experimental ICIDRI Masatepe.

Capacidades del CEIM. Como parte del desarrollo de este trabajo se identificaron fortalezas, oportunidades, debilidades y amenazas que presenta el CEIM, con la finalidad de que sea tomado en cuenta para el desarrollo de cualquier iniciativa que se pretenda realizar en un futuro tomando en cuenta los diversos procesos que demanda una investigación. A través de este Análisis FODA se encontraron aspectos relevantes que describen el estado de la finca y del circuito agro ecoturístico relacionados con los potenciales naturales y productivos identificados (Figura 2).

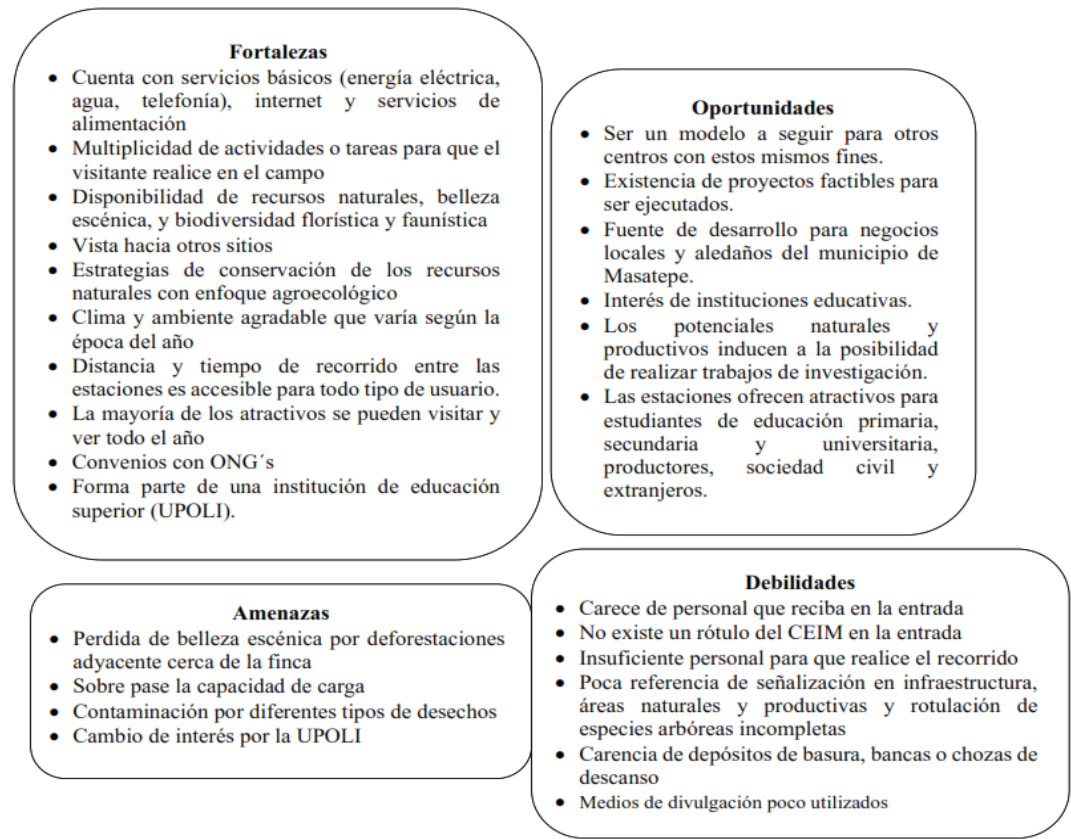

Figura 2. Análisis FODA del Centro Experimental ICIDRI Masatepe. 
A partir de las debilidades encontradas, se consideró oportuno elaborar estrategias básicas que son consensuadas por los miembros del ICIDRI para disminuir las debilidades, aprovechar las oportunidades, potenciar las fortalezas y controlar las amenazas (Cuadro 2).

Cuadro 2. Estrategias propuestas al Centro Experimental ICIDRI Masatepe para disminuir debilidades, aprovechar oportunidades, potenciar fortalezas y controlar las amenazas

\begin{tabular}{ll}
\hline \multicolumn{1}{c}{ Para disminuir debilidades } & \multicolumn{1}{c}{ Controlar las amenazas } \\
\hline - Construcción de caseta de seguridad en la & - Promover campañas locales de \\
entrada del centro con presencia de una persona & concientización para la conservación de los \\
que reciba y oriente al turístico y otros visitantes & recursos naturales \\
- Instalar señalización en la entrada del CEIM & - Realizar un estudio de capacidad de carga \\
y en área de Información (infraestructura, rutas & para llevar un control del número y visitas que \\
a seguir por los turistas y rutas de evacuación) & ingresen a los sitios \\
- Contratación de personal bilingüe y & - Disponer depósitos de basura en caminos \\
capacitado para atender a visitantes & principales para disminuir la presencia de \\
- Capacitación continua en aspectos de áreas & desechos sólidos presentes \\
experimentales, senderos interpretativos y de & \\
ecología-ambiente al personal para brindar un & \\
servicio de excelente calidad al visitante &
\end{tabular}

\begin{tabular}{|c|c|}
\hline Potenciar fortalezas & Aprovechar oportunidades \\
\hline $\begin{array}{l}\text { - Elaborar un descriptor (brochure o mapas de } \\
\text { información) con las potencialidades naturales y } \\
\text { productivas del CEIM para distintos usuarios } \\
\text { y/o escenarios educativos } \\
\text { - Realizar constantes mejoras e innovaciones } \\
\text { en la infraestructura y rotulaciones incompletas } \\
\text { - Promover y/o aprovechar los convenios con } \\
\text { otras ONG } \\
\text { - Desarrollar un plan de mantenimiento a los } \\
\text { sistemas productivos en conjunto con } \\
\text { colaboradores aliados }\end{array}$ & $\begin{array}{l}\text { - Divulgación del que hacer del CEIM en } \\
\text { conjunto con ICIDRI Managua y otras } \\
\text { instituciones que sirvan de alianza para } \\
\text { focalizar a diversos tipos de usuarios } \\
\text { - Establecer contactos con los negocios de la } \\
\text { localidad y zonas aledañas para la oferta de } \\
\text { sus productos } \\
\text { - Fortalecer relaciones con otras } \\
\text { organizaciones como universidades, ONG's, } \\
\text { instituciones de cooperación internacional, } \\
\text { entre otras, para el desarrollo de } \\
\text { investigaciones y capacitaciones en el CEIM }\end{array}$ \\
\hline
\end{tabular}

Propuesta de visitas y de contenidos temáticos para visitantes. La estrategia para implementar y ofrecer la propuesta del circuito agro ecoturístico del CEIM a los visitantes, se basa en el principio del trabajo conjunto entre el sector educativo del centro y de los intereses de los diversos tipos de visitantes. En el Cuadro 3, se proponen visitas por tipo de turistas.

La participación de los visitantes en actividades productivas es señalada por Barrera, citado por (Ramírez, 2013) como parte del agroturismo y se caracteriza porque el visitante es partícipe activo de las actividades dedicadas a la producción de cultivos y/o animales, y que éstas pueden ser con fines demostrativas o de producción. Por su parte Blanco y Riveros, citado por (Cutiupala, Baquero, Arévalo, Vinueza, y Velásquez, 2016) se refiere al agro ecoturismo como una herramienta de desarrollo integral impulsa la economía de los agricultores ya que integra las actividades tradicionales de producción como la agricultura y ganadería: a lo que se pretende incorporar la actividad alternativa del agro ecoturismo con lo que se mejorará la economía de una forma sostenible, impulsando los cultivos sanos, eficientes, rentables y competitivos y al mismo tiempo busca la satisfacción en los posibles visitantes.
Al relacionar este estudio con el de Cutiupala et al. (2016), se puede constatar la importancia de enfocar los potenciales de un sitio con la producción agrícola asociada al Cutiupala et al. (2016), turismo; y para asegurar la calidad del producto agro ecoturístico, se propone trabajar en áreas de acción (propuesta técnica para el desarrollo del producto agro ecoturístico) según el área que posee la Empresa, con el objetivo de mantener un control total y que a su vez permitan identificar los requerimientos en infraestructura, equipos y recorridos que necesitan para asegurar la calidad de servicios y actividades turísticas que se plantea ofertar a los visitantes.

En este sentido SECTUR MX, (2004) le denomina estaciones interpretativas y como contribución de este estudio para las explicaciones técnicas en cada parada o estación interpretativa se le agrega el término "temarios de contenido" para capacitar o desarrollar actividades (Cuadro 4).

Cuadro 3. Propuestas de visitas acordes a las estaciones y tipo de visitantes

\begin{tabular}{ll}
\hline \multicolumn{1}{c}{ Tipo de visitantes } & \multicolumn{1}{c}{ Propuestas de visitas acordes a las estaciones } \\
\hline Visitantes nacionales y & A este tipo de visitante se les propone las \\
extranjeros & estaciones 6 (La Ceiba), 7 (El Guanacastón) y 9 \\
& (El Mirador), donde pueden apreciar de manera \\
& general una exuberante belleza de flora y fauna; \\
& contar con espacios para la implementación de \\
& actividades recreativas.
\end{tabular}

Estudiantes de Para los estudiantes de colegios y universidades, educación secundaria y se recomienda visitar las estaciones 2 universitarios (Muestrario de Plantas Medicinales), 4 (Procesos del Café), 9 (El Mirador) y 10 (El Ensayo), donde recibirán información sobre el uso, importancia y formas de producción de las plantas medicinales, de los procesos del café, y el uso de camas Biointensivo con hortalizas.

Investigadores nacionales extranjeros Para despertar el espíritu de investigación se y recomiendan las estaciones 2 (Muestrario de Plantas Medicinales), 8 (Paso de las Palmeras), 10 (El Ensayo) y 11 (Cinco Pisos). En cada una de estas zonas, el investigador podrá, además, de participar en las diferentes actividades prácticas, considerar elementos claves que orienten a temas de investigaciones.

Productores Basado en los intereses para un grupo que demanda conocer nuevas experiencias técnicas en el campo y que requiere de espacios de capacitación, se proponen las estaciones 3 (Musáceas), 4 (Procesos del Café), 5 (Muestrario de Variedades de Café), 10 (El Ensayo) y 11 (Cinco Pisos), son las más apropiadas para ser consideradas como escuelas de campo por las prácticas implementadas. 
Cuadro 4. Temarios de contenidos acordes a las estaciones y tipo de visitantes

\begin{tabular}{|c|c|c|c|}
\hline Tema & Contenidos & Propuesta & Usuario \\
\hline \multirow{5}{*}{ Flora } & Tipos de hojas & $\begin{array}{l}\text { Identificación de los diferentes tipos de hojas } \\
\text { (compuestas y simples) en especies arbóreas y } \\
\text { su importancia ecológica }\end{array}$ & Estudiantes \\
\hline & Diversidad de fustes & $\begin{array}{l}\text { Características de las especies arbóreas por } \\
\text { tipos de fustes y de tallos, y su importancia } \\
\text { ecológica y económica }\end{array}$ & \multirow[t]{2}{*}{$\begin{array}{l}\text { Estudiantes y visitantes } \\
\text { nacionales, extranjeros }\end{array}$} \\
\hline & Frutos & $\begin{array}{l}\text { Tipos de frutos, el ciclo o periodo de floración } \\
\text { y fructificación (fenología) }\end{array}$ & \\
\hline & Variedad de especies & $\begin{array}{l}\text { Podrán conocer cuáles son las especies que } \\
\text { existen en el CEIM, identificar las diferencias } \\
\text { morfológicas }\end{array}$ & Estudiantes e investigadores \\
\hline & $\begin{array}{l}\text { Adaptación de las } \\
\text { especies }\end{array}$ & Especies exóticas existentes en el CEIM & Investigadores \\
\hline \multirow[b]{2}{*}{ Fauna } & Mi auto defensa & $\begin{array}{l}\text { Manera de auto defensa a depredadores } \\
\text { naturales y a factores de presión antrópicos }\end{array}$ & \multirow{2}{*}{$\begin{array}{l}\text { Estudiantes y visitantes } \\
\text { nacionales, extranjeros } \\
\text { Estudiantes y visitantes } \\
\text { nacionales, extranjeros e } \\
\text { investigadores }\end{array}$} \\
\hline & $\begin{array}{l}\text { ¿De qué me } \\
\text { alimento? }\end{array}$ & $\begin{array}{l}\text { ¿Cuál es la alimentación de estas especies? } \\
\text { periodo de alimentación y tiempo de } \\
\text { alimentación }\end{array}$ & \\
\hline \multirow{4}{*}{$\begin{array}{l}\text { Conservación de } \\
\text { suelo }\end{array}$} & $\begin{array}{l}\text { Microorganismos de } \\
\text { montañas }\end{array}$ & $\begin{array}{l}\text { Definición, importancia ecológica, forma de } \\
\text { producción, beneficios para la producción } \\
\text { agrícola y el suelo }\end{array}$ & \multirow{6}{*}{ Productores e investigadores } \\
\hline & Zanjas de infiltración & $\begin{array}{l}\text { Definición, importancia ecológica y } \\
\text { funcionamiento }\end{array}$ & \\
\hline & Camas Biointensivo & $\begin{array}{l}\text { Definición, importancia, construcción y } \\
\text { beneficios en la producción }\end{array}$ & \\
\hline & Abonos orgánicos & Importancia, procesos, aplicación y usos & \\
\hline \multirow{2}{*}{$\begin{array}{l}\text { Sistemas } \\
\text { agroforestales }\end{array}$} & $\begin{array}{l}\text { Sistemas } \\
\text { agroforestales }\end{array}$ & $\begin{array}{l}\text { Definición, importancia de los Sistemas } \\
\text { Agroforestales (SAF), cuáles son los SAF } \\
\text { implementados en el CEIM, beneficios de } \\
\text { estos para la producción }\end{array}$ & \\
\hline & Café bajo sombra & $\begin{array}{l}\text { Procesos para la producción del café, } \\
\text { preparación de biofertilizantes y fungicidas }\end{array}$ & \\
\hline $\begin{array}{l}\text { Educación } \\
\text { ambiental }\end{array}$ & Reciclaje & $\begin{array}{l}\text { Tipos, clasificación y reutilización de } \\
\text { desechos. Así mismo podrán realizar la } \\
\text { práctica de clasificación }\end{array}$ & $\begin{array}{l}\text { Estudiantes y visitantes } \\
\text { nacionales / extranjeros }\end{array}$ \\
\hline
\end{tabular}

\section{CONCLUSIONES}

El Centro Experimental Icidri Masatepe es un destino agro ecoturístico apto para ofrecer potenciales naturales y productivos; entre ellos: paisajes, avistamiento de aves, flora, fauna, un mirador donde se puede observar parches de bosque, procesamiento de café orgánico desde que está en plantaciones hasta que está lista para ser degustado, prácticas de preparación de biofertilizantes, fungicidas orgánicos y otros sistemas productivos.

De acuerdo con la descripción de las capacidades del centro, entre las fortalezas que se destacan están: la implementación de estrategias de conservación de los recursos naturales con enfoque agroecológico, las diversas actividades de campo que promueven la participación de visitantes (estudiantes, investigadores y productores).

En los aspectos negativos: insuficiente personal para recibir a los visitantes en la entrada y para el recorrido de las estaciones, carece de depósitos de basura en caminos principales para disminuir la presencia de desechos sólidos presentes y poca señalización. Pero como aspecto de mayor urgencia es promover la divulgación, la investigación y el intercambio de experiencias entre los diversos tipos de usuarios para campar visitantes y desarrollar sus potencialidades.

Basado en las condiciones del sitio, potenciales naturales y productivos e interés del centro, se puede establecer un sendero interpretativo en forma de circuito, siendo este muy útil para visitantes nacionales y extranjeros, entre ellos estudiantes, investigadores y productores.

El CEIM es por tanto un reservorio natural en una zona rural donde se pueden desarrollar encuentros entre técnicos, investigadores, productores y practicas estudiantiles a nivel básico como superior. Todas y cada una de las experiencias que se pueden promover pueden contribuir con la promoción de nuevas tecnologías educativas y tecnológicas. 


\section{REFERENCIAS BIBLIOGRÁFICAS}

Blanco, M. (2008). Guía para la elaboración del plan de desarrollo turístico de un territorio. IICA. San José, Costa Rica. http://www. territorioscentroamericanos.org/experiencias/Documents/Costa\%20Rica/M.\%20Gu\%C3\%ADa\%20turismo\%20territorio.pdf.

Castillo Conde, C. M. (2017). Importancia del desarrollo turístico del circuito vivencial amazonas y su influencia en la economía de la región - 2017. Facultad de Ciencias de La Comunicación Turismo y Psicología. Escuela de Turismo y Hotelería. (pp. 45-47). http:// www.repositorioacademico.usmp.edu.pe/bitstream/handle/usmp/3399/castillo_ccm.pdf?sequence=3\&isAllowed=y

Cutiupala, G. M. A., Baquero, J. C. C., Arévalo, P. A. V., Vinueza, S. A. B., \& Velásquez, C. R. C. (2016). Diseño De Un Producto AgroEcoturístico En La Propiedad De La Empresa Agropecuaria Y Turística “ A Y T” Parroquia San Isidro, Cantón Guano, Provincia De Chimborazo. European Scientific Journal, ESJ, 12(32), 146. https://doi.org/10.19044/esj.2016.v12n32p146ICIDRI-UPOLI. (2011). Planificación Estratégica del Centro Experimental ICIDRI Masatepe. Nicaragua.

Instituto Nicaragüense de Turismo. (2009). Definición de politicas y estrategias para el Turismo Rural Sostenible de Nicaragua. Informe país por Sergio Santamaria Zapata y Roberto Emilio Baca. Baca. 116 p.

Larios-González, R. C., Salmerón-Miranda, F., y García-Centeno, L. (2014). Fertilidad del suelo con prácticas agroecológicas y manejo convencional en el cultivo de café. La Calera, 14(23). https://lacalera.una.edu.ni/index.php/CALERA/article/view/211/211

López Acevedo, J. E. y Ráudez Mairena, I. N. (2010). Ecoturismo Comunitario en diez fincas del Parque Ecológico Municipal Canta Gallo, Condega-Esteli (tesis de pregrado). Universidad Nacional Agraria, Managua, Nicaragua.

Mérida, G., García, W., Vásquez, E., y Oros, R. (2003). Agroturismo y ecoturismo en Colomi: una estrategia para la conservación in situ de la biodiversidad. En W., García y X, Cadina (Ed). Manejo sostenible de la agrobiodiversidad de tubérculos andinos: síntesis de investigaciones y experiencias en Bolivia. (pp. 187-197). https://books.google.com.ni/

Ramírez Castellano, E. D. (2015). Agro ecoturismo: Aportes Para El Desarrollo De Una Tipología Turística En El Contexto Latinoamericano. Universidad de la Salle, Colombia, 14 p.

Ramírez Castellano, E. D. (2013). Agro ecoturismo: aportes para el desarrollo de una tipología turística en el contexto latinoamericano. Anuario Turismo y Sociedad, XV, 223-236. DOI: 10.18601/01207555.n15.13

Redclift, M. y Woodgate, G. (1993). Concept of the Environment in the Social Sciences. Wye college External programme. Wye England.

Riveros, H., y Blanco, M. (2003). El agroturismo, una alternativa para revalorizar la agroindustria rural como mecanismo de desarrollo local, Lima, PE, IICA-PRODAR, 33 p.

Sáenz Freire, J. A. (2017). Análisis del potencial desarrollo productivo especializado en agro- ecoturismo en la "hacienda el quetzal" recinto de las Tolas, parroquia Gualea, cantón quito, provincia de pichincha. Universidad católica del ecuador. Facultad de ciencias humanas escuela de hotelería y turismo. (pp. 21). http://repositorio.puce.edu.ec/bitstream/handle/22000/13234/ AN\%C3\%81LISIS\%20DEL\%20POTENCIAL\%20DESARROLLO \%20PRODUCTIVO \%20ESPECIALIZADO \%20EN\%20 AGRO-\%20ECOTURISMO $\% 20 \% 281 \% 29$.pdf? sequence=1\&isAllowed=y

Secretaría de Turismo de México. (2004). Guía para el diseño y operación de senderos interpretativos. México. https://www.academia. edu/36598500/Gu\%C3\%ADa_para_el_dise $\%$ C3\%B1o_y_operaci\%C3\%B3n_de_senderos_interpretativos

Vaca Viteri, M. S. (2001). Circuito Turístico Guayaquil-Salinas-Puerto Cayo (tesis pregrado). Recuperado de http://www.dspace.espol. edu.ec/bitstream/123456789/4615/1/7136.pdf 